Ida Dielemans

Johannes R. Vingerling

Albert Hofman

Diederick E. Grobbee

Paulus T. V. M. de Jong

\section{Reliability of intraocular pressure measurement with the Goldmann applanation tonometer in epidemiological studies}

Received: 28 June 1993

Revised version received: 2 September 1993

Accepted: 28 October 1993

I. Dielemans · J. R. Vingerling

P. T. V. M. de Jong

Department of Ophthalmology, Erasmus

University Medical School, Rotterdam,

The Netherlands

I. Dielemans $\cdot$ J. R. Vingerling

A. Hofman - D. E. Grobbee

Department of Epidemiology and

Biostatistics, Erasmus University Medical

School, Rotterdam, The Netherlands

P. T. V. M. de Jong ( $ه$ )

Department of Ophthalmology,

Academic Hospital Dijkzigt,

Dr. Molenwaterplein 40, NL-3051 GD

Rotterdam, The Netherlands

\begin{abstract}
The reproducibility of intraocular pressure (IOP) measurement with the Goldmann applanation tonometer was investigated as part of a population-based epidemiological study. Sixty-two subjects were examined in a first measurement session. The IOP was measured three times consecutively in both eyes according to a fixed protocol. The mean standard deviation (SD) of these measurements was $0.8 \mathrm{mmHg}$. The mean intraobserver variation for the first measurement was 1.64 (SD 2.07) $\mathrm{mmHg}$. For the median of the three measurements the intra-ob-
\end{abstract}

server variation was 1.50 (SD 1.96) $\mathrm{mmHg}$. The mean inter-observer values were 1.79 (SD 2.41) $\mathrm{mmHg}$ for the first measurement and 1.60 (SD 2.15) $\mathrm{mmHg}$ for the median measurement. The correlation coefficient between the median values of the three measurements of both observers was 0.81 . No systematic differences were found between the two observers. Using the median value of three consecutive measurements reduced the inter-observer variation by $11 \%$ and the intra-observer variation by $9 \%$ compared with a single measurement.

\section{Introduction}

The Goldmann tonometer for measurement of intraocular pressure (IOP) has been in use since 1957 [5]. It is the accepted standard in ophthalmology $[5,14,21]$. In comparison with other measurement techniques, the Goldmann device is thought to give more accurate results over a wider range of IOP values $[4,10,19,21]$. In practice, however, there is intra- and inter-observer variation $[3,12,15,16,18,24]$. IOP in epidemiological studies has been defined in many different ways (Table 1). In most studies no validation for these techniques has been given. In order to check the reliability of IOP measurements in an epidemiological study we compared the reproducibility of the median of three consecutive measurements with a single measurement of IOP with the Goldmann applanation tonometer.

\section{Subjects and methods}

\section{Subjects}

Sixty-two persons out of the first 1000 randomly selected participants of the Rotterdam Study participated in this study [7]. The Rotterdam Study is a population-based follow-up study of 11854 people aged 55 years or over, concentrating on chronic disabling diseases in the elderly, including glaucoma and age-related macular degeneration. The study protocol had been approved by the medical ethical committee of the Erasmus University. The validation study of IOP measurement consisted of two parts.

In the inter-observer variation study, 17 men and 23 women, consecutive participants of the Rotterdam Study, were included. Their mean age was 69.6 years (SD 7.7 years) and they had no corneal abnormalities. The IOP of first the right and then the left eye of each subject was taken three times by one observer. After 10 min a second observer took a similar series of three measurements of each eye. Ten minutes was arbitrarily chosen as the interval between the two measurement series in order not to keep the subjects waiting too long. Both observers were well trained and had taken applanation IOPs in over 4000 subjects. The sequence of the observers was random. 
In the intra-observer study, an additional 22 consecutive participants of the Rotterdam Study, in the same age group as in the first part of the study (mean age 69.6 years, SD 6.6 years) and also without corneal abnormalities were examined, using the same protocol. The only difference was that IOP measurements were repeated after 10 min by the same observer.

\section{Study protocol}

All readings were obtained with the same Goldmann tonometer (Haag-Streit, Bern, Switzerland), mounted on a Haag-Streit 900 BM slit lamp with a standard blue filter. The calibration of the tonometer had been checked just before the study.

The protocol for IOP measurement was as follows. One drop of oxybuprocaine $0.4 \%$ was instilled into each eye of the subject. Both inferior conjunctival sacs were touched with a dry fluorescein strip (Haag-Streit). Before each measurement the scale of the tonometer was set at $10 \mathrm{mmHg}$. If necessary, the upper eyelid was lifted by rotating a cotton-tipped stick against the orbital rim, taking great care to avoid compression of the globe. The tip of the tonometer was illuminated with a wide-open slit at an angle of 45 deg. The applanating prism, with its axis on $0 \mathrm{deg}$, was lightly pressed against the center of the cornea. In the case of a marked corneal astigmatism resulting in an elliptical rather than a circular contact area, the prism must be rotated so that the dividing line lies at about $45 \mathrm{deg}$ to the major axis of the ellipse [5]. The tonometer was raised or lowered in such a position that both semicircles were of equal size. Care was taken that the menisci were of equal circumference and of appropriate width, namely, approximately 0.05 th part of the outer diameter of the semicircle. Strictly without looking at the dial, the rotating knob of the tonometer was set at a level in such a way that the inner aspect of the two mires just touched. If the IOP fluctuated during the cardiac pulse cycle, the measurement was taken at mid-cycle. If the bar on the rotating dial touched a fixed bar of the tonometer, that pressure was noted. If the movable bar was free of the fixed bars, the intermediate pressure was taken. Subsequently, the dial of the tonometer was set again at $10 \mathrm{mmHg}$. In this way, three consecutive measurements were performed on each eye and each value was recorded immediately after the measurement. The right eye was always measured first.

\section{Data analysis}

The data were analyzed by means of determination of standard deviations and coefficients of variation of three consecutive IOP measurements and of differences between the median values of the two observers. The coefficient of variation was defined as the standard deviation divided by the mean value of the three consecutive IOP measurements. Correlation coefficients of the median values of the two observers were determined. The paired standard deviations of the three measurements of individual cases were compared between the observers using Wilcoxon's test. Median values of the three consecutive measurements were compared between and within the observers using paired $t$-tests [6]. All $P$ values given are two-sided, with 0.05 taken as the level of significance.

\section{Results}

In the inter-observer study, 40 right and 39 left eyes (one subject wore a prosthesis) were examined. The differences between three consecutive IOP measurements taken by one observer varied between 0 and $5 \mathrm{mmHg}$.
Table 1 Methods of defining intraocular pressure in validation studies and epidemiological studies using applanation tonometry with the Goldmann tonometer

\begin{tabular}{|c|c|}
\hline Method & Reference(s) \\
\hline First single measurement value & $1,3,11,12,15,19$ \\
\hline $\begin{array}{l}\text { Taking measurements until } \\
\text { three successive readings were } \\
\text { within } 1 \mathrm{mmHg} \text { of each other }\end{array}$ & 16,18 \\
\hline Mean of three consecutive readings & 9 \\
\hline Mean of two consecutive readings & 24 \\
\hline Median of three consecutive readings & 23, this paper \\
\hline No description & $2,8,13,22$ \\
\hline
\end{tabular}

Table 2 Difference between first and median IOP measurements $(\mathrm{mmHg})$ between and within observers

\begin{tabular}{lll}
$\begin{array}{l}\text { First } \\
\text { measurement } \\
\text { (A) }\end{array}$ & $\begin{array}{l}\text { Median of } \\
\text { three measurements }\end{array}$ & Difference \\
(B) & (A-B) \\
\hline
\end{tabular}

Difference between observers ( $n=79$ eyes of 40 subjects $)$

$\begin{array}{llll}\text { Mean } & 1.79 & 1.60 & 0.19(11 \%) \\ \text { SD } & 2.41 & 2.15 & 0.26(11 \%)\end{array}$

Difference within observers ( $n=44$ eyes of 22 subjects)

\begin{tabular}{llll} 
Mean & 1.64 & 1.50 & $0.14(9 \%)$ \\
$\mathrm{SD}$ & 2.07 & 1.96 & $0.11(5 \%)$ \\
\hline
\end{tabular}

The SDs of these three measurements for the right eye were $0.7 \mathrm{mmHg}$ for both observers (coefficient of variation, $\mathrm{CV}, 5 \%$ ). For the left eye the SD was $0.8 \mathrm{mmHg}$ (CV 6\%) for observer $\mathrm{A}$ and $0.9 \mathrm{mmHg}(\mathrm{CV} 7 \%$ ) for observer B. The mean of these four SDs was $0.8 \mathrm{mmHg}$. The level and variability of the IOP readings were independent of the age and gender of the subjects.

The correlation coefficient of the median values for the two observers was 0.75 for the right eye and 0.87 for the left eye. Taking the mean of the values of both eyes, the correlation coefficient was 0.81 . The difference between the median values of the two observers had a mean of $1.60 \mathrm{mmHg}$ (SD $2.15 \mathrm{mmHg}$ ). The difference between the first measurements of the two observers had a mean of $1.79 \mathrm{mmHg}$ (SD $2.41 \mathrm{mmHg}$ )(Table 2).

Comparing median values of the three consecutive IOP readings between the two observers, the second observer, $10 \mathrm{~min}$ after the first, measured lower values. The average decrease was $0.7 \mathrm{mmHg}$ (standard error 0.4 $\mathrm{mmHg}, P=0.07$ ) for the right and $0.5 \mathrm{mmHg}$ (standard error $0.2 \mathrm{mmHg}, P=0.04$ ) for the left eye. Figure 1 shows the measured IOP at both occasions for both eyes.

In the intra-observer study, both eyes of an additional 22 subjects were examined. The mean difference between the median values was $1.50 \mathrm{mmHg}$ (SD 1.96 $\mathrm{mmHg}$ ). The mean difference between the first measure- 

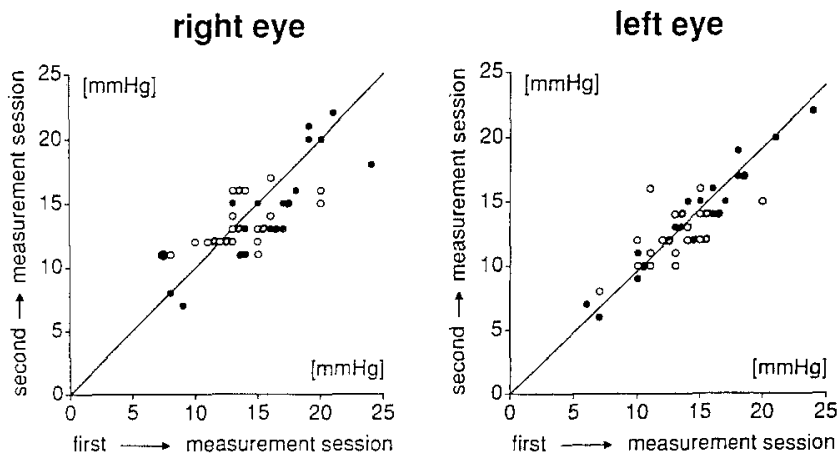

Fig. 1 Intraocular pressure (IOP) in first and second measurement sessions. The line denotes identity. Each IOP represents the median value of three measurements $(n=40)$. Closed and open circles indicate subjects in whom the first measurement was done by the first and second observer, respectively

ments of the series of three was $1.64 \mathrm{mmHg}$ (SD 2.07 $\mathrm{mmHg}$ ) (Table 2). The average decrease of the median values at the second measurement session was 0.9 $\mathrm{mmHg}$ for both eyes $(\mathrm{P}<0.01)$.

\section{Discussion}

The findings indicate that a protocol using the median of three readings of IOP measurement with the Goldmann tonometer gives better reproducibility of the IOP than single measurements. In comparison with the first single IOP measurement the inter-observer variation decreased by $11 \%$ and the intra-observer variation by $9 \%$. For IOP measurements in a glaucoma clinic this may seem to be a small improvement, given the fact that nowadays more emphasis is put on visual field and optic nerve head evaluation than on absolute values of IOP. However, in epidemiological studies, where differences in IOP of $1-3 \mathrm{mmHg}$ may point to an increased risk of glaucoma, there is a need for reproducible and precise measurement techniques.

Because one IOP measurement is subject to variation [16], it was decided to perform three consecutive measurements to obtain a better estimate of the actual IOP. The SD of the three consecutive IOP measurements at one occasion by one observer on one eye was $0.8 \mathrm{mmHg}$ in this study. This figure is similar to that from a study by Thorburn $(0.9 \mathrm{mmHg})$ in which two consecutive measurements were made by the same observer [24].
However, in other studies this $\mathrm{SD}$ amounted to $3 \mathrm{mmHg}$ [16] and $4.07 \mathrm{mmHg}$ [20].

A fixed number of consecutive measurements was chosen because each subsequent measurement lowers the IOP by $0.1-0.4 \mathrm{mmHg}[16,17]$. These values fit within the $0.5-0.7 \mathrm{mmHg}$ difference that were found in this study after $10 \mathrm{~min}$. Medians were used instead of mean values in order to reduce the influence of outlying values. The correlation coefficient of the two observers' median values was quite high (0.81) in this study. In a study with a variable number of measurements [18], the correlation coefficient was 0.71 .

The standard deviation of the inter-observer differences in the present study was $2.15 \mathrm{mmHg}$, similar to the value of $2.5 \mathrm{mmHg}$ given in the study of Phelps and Phelps [18]. The SD of the intra-observer differences was $1.96 \mathrm{mmHg}$ in this study, which is similar to the value of $1.4 \mathrm{mmHg}$ found by Moses and Liu [16].

In this study a fixed protocol of IOP measurement was used to standardize effects of factors known to influence the result, such as the use of fluorescein. Applanation tonometry without the use of fluorescein resulted in a mean IOP value $5.62 \mathrm{mmHg}$ lower than applanation tonometry with fluorescein use [20]. A dry fluorescein strip was used, not a mixture of fluorescein with a local anesthetic drop, as the former was used in the original calibration technique of the Goldmann tonometer [5] and resulted in a mean IOP value $3.94 \mathrm{mmHg}$ lower than the latter [20]. The menisci were of equal circumference and of appropriate width when the measurement was made, and the measurement was taken in mid-cycle $[5,14,18,20]$.

In conclusion Goldmann applanation tonometry readings varied by $6 \%$ among three measurements. The present study suggests that the reliability of IOP measurement with the Goldmann tonometer may be moderately improved by using the median of three consecutive IOP measurements rather than relying on one measurement.

Acknowledgements The work reported here was supported by the Nestor stimulation programme for geriatric research in The Netherlands (Ministry of Health and Ministry of Education); Topcon Europe BV.; Haags Oogheelkundig Fonds; Merck, Sharp \& Dohme - Chibret Nederland; Stichting Bevordering van Volkskracht; Stichting Blindenhulp; Rotterdamse Vereniging voor Blindenbelangen; and The Netherlands Society for the Prevention of Blindness. We wish to thank Wim Hop for his statistical support and the co-workers of the Rotterdam Study, in particular Els Baars, Diana Bakker, Ada Hooghart, Anneke Korving, Peter Boerlage and Ronald Stolk, for their help.

\section{References}

1. Bechrakis $E$ von (1966) Über den spontanen Druckabfall bei Applanation-Tonometrie. Ophthalmologica 151:604-614
2. Bengtsson $\mathrm{B}$ (1981) The prevalence of glaucoma. Br J Ophthalmol 65:46-49
3. Berry V, Drance SM, Wiggins RL, Schulzer M (1966) A study of the errors of applanation tonometry and to. nography on two groups of normal people. Can J Ophthalmol 1:213-220 
4. Frenkel REP, Hong YJ, Shin DH (1988) Comparison of the Tono-Pen to the Goldmann applanation tonometer. Arch Ophthalmol 106:750-753

5. Goldmann H, Schmidt T (1957) Über Applanationstonometrie. Ophthalmologica 134:221-242

6. Hills M, Armitage P (1979) The twoperiod cross-over clinical trial. $\mathrm{Br} \mathrm{J}$ Clin Pharmacol 8:7-20

7. Hofman A, Grobbee DE, de Jong PTVM, van de Ouweland FA (1991) Determinants of disease and disability in the elderly: The Rotterdam Elderly Study. Eur J Epidemiol 74:403-422

8. Hollows FC, Graham PA (1966) Intra-ocular pressure, glaucoma, and glaucoma suspects in a defined population. Br J Ophthalmol 50:570-586

9. Kahn HA, Leibowitz HM, Ganley JP, Kini MM, Colton T, et al. (1977) The Framingham Eye Study. 1. Outline and major prevalence findings. Am J Epidemiol 106:17-32

10. Kao SF, Lichter PR, Bergstrom TJ, Rowe S, Musch DC (1987) Clinical comparison of the Oculab Tono-Pen to the Goldmann applanation tonometer. Ophthalmology 94:1541-1544
11. Klein BEK, Klein R, Sponsol WE, Franke T, Cantor LB, et al. (1992) Prevalence of glaucoma. The Beaver Dam Eye Study. Ophthalmology 99:1499-1504

12. Krakau CET, Wilke K (1971) On repeated tonometry. Acta Ophthalmol 49:611-614

13. Leske MS, Connell AMS, Kehoe R (1989) A pilot project of glaucoma in Barbados. Br J Ophthalmol 73:365369

14. Moses RA (1958) The Goldmann applanation tonometer. Am J Ophthalmol 46:865-869

15. Moses RA (1961) Repeated applanation tonometry. Ophthalmologica 142:663-668

16. Moses RA, Liu CH (1968) Repeated applanation tonometry. Am J Ophthalmol 66:89-91

17. Motolko MA, Feldman F, Hyde M, Hudy D (1982) Sources of variability in the results of applanation tonometry. Can J Ophthalmol 17,3:93-95

18. Phelps CD, Phelps GK (1976) Measurement of intraocular pressure: a study of its reproducibility. Graefes Arch Clin Exp Ophthalmol 198:39-43
19. Quigley HA, Langham ME (1975) Comparative intraocular pressure measurements with the pneumotonograph and Goldmann tonometer. Am J Ophthalmol 80:266-273

20. Roper DL (1980) Applanation tonometry with and without fluorescein. Am J Ophthalmol 90:668-671

21. Shields MB (1982) The intraocular pressure. In: Shields MB (ed) A study guide for glaucoma. Williams \& Wilkins, Baltimore, pp 56-60, 141-143, 163

22. Shiose Y, Komuro K, Itoh T, Amano M, Kawase Y (1981) New system for mass screening of glaucoma, as part of automated multiphasic health testing services. Jpn J Ophthalmol 25:160-177

23. Tielsch JM, Sommer A, Katz J, Royall RM, Quigley HA, et al. (1991) Racial variations in the prevalence of primary open-angle glaucoma. The Baltimore Eye Survey. JAMA 266:369-374

24. Thorburn W (1978) The accuracy of clinical applanation tonometry. Acta Ophthalmol 56:1-5 